\title{
WORKING GROUP ON Ap AND RELATED STARS
}

\section{(GROUPE DE TRAVAIL POUR LES ETOILES PARTICULIERES DE TYPE A)}

\author{
CHAIRPERSON: Masahide Takada-Hidai \\ MEMBERS: Georges Alecian, Richard O. Gray, Marco Landolf, \\ Pierre North, Kazimierz Stepien, Werner W. Weiss \& Juraj Zverko
}

\section{CONFERENCES}

\subsection{Joint Discussion 16 at the 23rd IAU General Assembly, Kyoto, Japan}

The JD 16 was held on August 25, 1997 as one-day session with the title of "Spectroscopy with Large Telescope of Chemically Peculiar Stars". It was supported by the Division IV and Commissions 14, 27, 29, 36, and 45.

The members of SOC were: G. Alecian, J. Babel, M. Gerbaldi, R. O. Gray, G. Mathys, P. North, M. Takada-Hidai (Chairperson), and J. Zverko (Co-chairperson).

15 talks and 18 contributed posters were presented on six topics of (1) model atmospheres and atomic data, (2) stellar parameters, (3) LTE and NLTE abundance analyses, (4) diffusion processes, (5) magnetic fields, and (6) variabilities. Approximate number of participants amounted to 50 at maximum.

The talks were published in Highlights of Astronomy (Andersen, 1998).

\subsection{The 26th Meeting and Workshop of the European Working Group on CP stars}

The meeting was held in Vienna on October 27-29, 1997. The members of SOC were; G. Alecian, J. Babel, F. Castelli, M. Gerbaldi, H.M. Maitzen, P. North (Chairperson), W.W. Weiss, and J. Ziznovsky. The LOC were; H.M. Maitzen, E. Paunzen, M. Rode, A. Schnell (Chairperson), W.W. Weiss.

There were 74 participants from 21 countries. Invited and contributed talks and posters were presented on Hipparcos results, roAp stars, Doppler imaging of magnetic Ap stars, and some results on lambda Boo stars.

An evening lecture by Dr. Anneliese Schnell, entitled "100 years ago", described the work and life of Antonia Maury (discoverer of the first Ap star in 1897), of other "Harvard ladies" and the education and careers of women in general at that time.

All contributions were published in the Contrib. Astron. Obs. Skalnate Pleso Vol. 27, No 3 (April 1998).

\section{Ap NEWSLETTER}

The Ap Newsletter (APN) was originally founded in 1978 for the purpose to exchange information of Ap star studies. It has appeared (electronically) twice a year since March 1995 , when P. North became an editor. Five issues of APN with No. $27-31$ have been distributed since 1997.

Since October 1998, there has been a "working issue" of APN in addition to the "published" one: this working issue is simply a provisional draft of the next issue, where 
the abstracts are included almost as soon as they are received, and it is made public on the web. Then it is cristallized 6 months later. This allows the abstracts to be visible rapidly, before the corresponding paper is published, while maintaining the low frequency of 2 "published" issues per year.

Recent issues treated topics on Ap stars as well as other related stars: CP 1 - 4 stars, lambda Boo stars, winds in A and B stars, field horizontal branch stars and objects possibly related to barium stars, as well as spectroscopy in general.

Editor of APN: Pierre NORTH (1997 Aug. - 2000 Aug.)

Acknowledgments. I would like to express my thanks to P. North and J. Zverko for their help.

\author{
Masahide Takada-Hidai \\ Chairperson of the Working Group
}

\title{
References
}

Highlights of Astronomy, 11B, 645 (ed.) J. Andersen, Kluwer Academic Publishers, Dordrecht 1998.

Proceedings of the 26th Meeting and Workshop of the European Working Group on CP stars, ed. P. North, A. Schnell, J. Žižňovský, Contrib. Astron. Obs. Skalnate Pleso Vol. 27, No 3, p.144 (1998). 